psychiatric emergency service, six patients manifested a Capgras delusion; four of these patients presented with the chief complaint of violence towards a family member (Fishbain, 1987). Only on inquiry as to the reason for the attack did the Capgras delusion become evident. Such observations indicate that unless specific inquiry is undertaken for a misidentification syndrome, the diagnosis could be missed, especially if the patient manifests additional delusional material. Violence or threatened violence towards family members is a relatively common psychotic presentation (Benezech et al, 1980). These findings and observations have led me to postulate the following: misidentification syndromes could be responsible for a much greater percentage of psychotic violent acts towards significant others and/or family members than once thought, but this diagnosis is routinely missed (Fishbain, 1987). I have therefore suggested that a chief complaint of violence towards a significant other and/or family member in a psychotic patient should alert the examining psychiatrist to the possibility of a misidentification syndrome.

Comprehensive Pain and Rehabilitation Center David A. Fishbain

\section{Alton Road}

Miami Beach

Florida 33139, USA

\section{References}

Fishrain, D. A. (1987) The frequency of Capgras delusions in a p: chiatric emergency service. Psychopathology, 20, 42-47.

Bent 7eCh, M., Bourgeois, M. J. \& YesA VAGE, J. (1980) Violence in the mentally ill: a study of 547 patients at a French hospital for the criminally insane. Journal of Nervous and Mental Disease, 168, 698-700.

The Need to Compare the Effectiveness of Antidepressant Drugs: In Which Patient Populations?

SIR: The analysis of the present state of knowledge and its inherent limits in the field of antidepressant agents made by Garattini (Journal, January 1988, $152,140-141)$ is realistic and has important consequences. In this therapeutic area, old and new compounds can be satisfactorily described in terms of their pharmacological activities (more than in terms of their mechanisms of action), but our understanding of the clinical relevance of their properties as to their efficacy is nonexistent.

The ex-juvantibus approach utilised to try to understand and describe the biological basis of depressive disorders through psychopharmacology has favoured the growth of scientific knowledge, but seems now to have come to a standstill: at present, it seems highly unlikely that any abnormality of a single biochemical system possibly cietected in this way could be related to a nosological entity (van Praag, 1986).

As we know, hypotheses can only be disproved, and we should search for differences; Dr Garattini suggests seeking differences by increasing the sample size of comparative clinical trials or by focusing on non-responders. On the other hand, differences within the patient populations studied as to their diagnostic classification, the clinical prerequisite (Ban, 1987), could be similarly important.

Acquisition of new knowledge in pharmacological research depends upon a process that "symmetrically relates diagnosis, treatment and outcome" (Joyce, 1986). The available diagnostic instruments have been shown to be able, at the most, to discriminate therapeutically more responsive patient populations from therapeutically less responsive ones, thus providing sufficiently homogeneous samples for establishing the efficacy of antidepressant drugs; the use of diagnostic instruments adequate to identify diagnostically distinct patient sub-populations would allow a more efficient detection of the differences between drugs. Identification and validation of more sensitive diagnostic approaches would provide reliable methods to collect the information needed for new and, perhaps, more productive efforts of pharmacological research. Proposals are already available (Ban, 1987).

Farmitalia Carlo Erba, $R \& D, C N S$ Line

Adriana Dubini

Via Imbonati, 24

20159 Milano

Italy

\section{References}

BAN, T. (1987) Prolegomenon to the clinical prerequisite: psychopharmacology and the classification of mental disorders. Progress in Neuro-Psychopharmacology and Biological Psychiatry, $11,527-580$.

JoYCE, C. R. B. (1986) New drugs for mental diseases? Journal of the Royal Society of Medicine, 80, 406-409.

van Prang, H. M. (1986) The role of biological psychiatry in psychiatry. In Biological Psychiatry in Europe Today (eds L. Pepplinkhuizen and W. M. A. Verhoeven). Leiderdorp: Uitgeverij De Medicus.

\section{Barking mad}

SIR: Recently I was asked to see an 86-year-old lady who had developed a paranoid illness for the first time following a prolonged hospital stay. Ward staff were bewildered by her occasional barking at night and, I am afraid, I was unable to enlighten them in relation to this matter. As the patient did have a delusional persecutory system, I suggested that she be started on thioridazine. 